\title{
Oral contraceptive use and salivary C-erbB-2, CEA and CA15-3 in healthy women: A Case-Control Study
}

\author{
Mohammad Vahedi ${ }^{1}$, Shermin Abdollahzadeh ${ }^{1}$, Pejman-Bakianian Vaziri ${ }^{2}$, Hamed Mortazavi ${ }^{1}$ \\ ${ }^{1}$ Assistant professor, Dep. Of Oral Medicine, Dental School, Hamadan University of Medical Sciences, Hamadan, Iran \\ ${ }^{2}$ Assistant professor, Dep. Of Oral Medicine, Dental School, Shaheed Beheshti University of Medical Sciences, Tehran, Iran
}

\author{
Correspondence: \\ Oral Medicine Department, \\ Shaheed Fahmideh Boulevard, \\ In Front of Mardom Park, Dental School, \\ Hamadan University of Medical Sciences, \\ Hamadan, Iran. \\ P. O. Box 65178-3-8736, Hamadan, Iran \\ Shery13562002@yahoo.com
}

Received: $11 / 11 / 2009$

Accepted: 30/03/2010
Vahedi M, Abdollahzadeh S, Vaziri PB, Mortazavi H. Oral contraceptive use and salivary C-erbB-2, CEA and CA15-3 in healthy women: A CaseControl Study. Med Oral Patol Oral Cir Bucal. 2011 Jan 1;16 (1):e29-32. http://www.medicinaoral.com/medoralfree01/v16i1/medoralv16ilp29.pdf

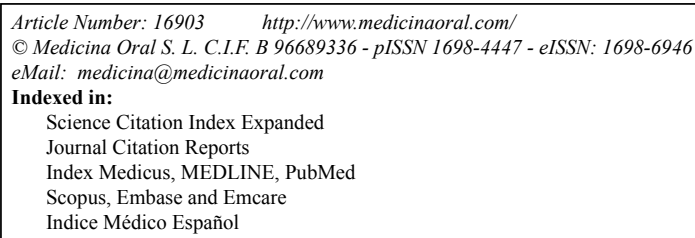

\begin{abstract}
Objectives: Oral contraceptives (OCP) are highly effective, safe and widely used. Higher exposure to endogenous and exogenous estrogens is generally thought to increase the risk of breast cancer. Therefore, this study was conducted to determine if oral contraceptive use affected the expression of CA 15-3, CEA and C-erb B-2 in the saliva of healthy women.

Study design: The participants consisted of 87 healthy women (43 controls and 44 using oral contraceptives) ranging in age from 20 to 54 years. The volunteers participated by giving one - time stimulated whole saliva samples. Then the samples were analysed for CA 15-3, CEA and C-erb B-2 concentrations.

Results: The student t-test was used to compare group means for variables with comparable variability. The mean of C-erb B-2, CEA, and CA 15-3 concentrations (in the case and control groups) was $(1.93,1.70),(34.46,31.62)$ and $(12.58,16.19)$ respectively. These differences were not statistically significant.

Conclusions: Our findings suggest that the levels of the cancer biomarkers C-erb B-2, CEA and CA 15-3 were not affected by increased levels of estrogens in the body.
\end{abstract}

Key words: Saliva, oral contraceptive, breast cancer, C-erb B-2, CEA, CA 15-3.

\section{Introduction}

Oral contraceptives (OCP) are highly effective, safe and widely used, as $80 \%$ of all 35 years old women use or have used OCP in their lifetime. Side effects are the most common reported reason why women discontinue using OCP $(1,2)$. There is considerable evidence that the hormone replacement therapy is more strongly associated with the risk of some invasive breast carcinomas (3). The incidence of breast cancer in 2007 may have decreased from previous decades but among women worldwide breast cancer is the most common cause of cancer death $(4,5)$. Attention to breast cancer screening, risk assessment and risk reduction strategies is necessary to eradicate breast cancer and the deaths it causes (4). Saliva plays an important role in the protection of oral cavity and alterations in either salivary flow rate or protein composition may have dramatic effects on oral health. Diagnosis of diseases via the analysis of saliva is 
potentially valuable since collection of the saliva is associated with fewer compliance problems as compared with the collection of blood samples (5). Three biomarkers that have been reported to be linked with breast cancer are C-erbB-2, CEA and CA15-3. C-erbB-2 (Her2/ neu) is a proto-oncogen localized to chromosome $17 \mathrm{q}$ and encodes a transmembrane tyrosine kinase growth factor receptor. The over expression of Her $2 /$ neu has been associated with breast, colorectal and head and neck cancers $(6,7)$. The soluble $\mathrm{C}$-erbB-2 receptor protein levels have been detected in serum, nipple aspirates and saliva (7). It has been reported that the assay of salivary C-erbB-2 protein is reliable and might have potential use in the initial detection and follow up screening of breast cancer (8). CA15-3, a high-molecular-mass mucin-like glycoprotein, is expressed at the luminal surface of most secretary epithelial (9). This marker has been added to the recommendations of American Society of Clinical Oncology for use in tumor marker test panels (2). Currently the main clinical application of CA15-3 is for monitoring the patients with diagnosed breast carcinoma and preclinically detecting recurrences (7). It was also shown that the levels of circulating CA15-3 correlated with tumor size, thus reflecting disease stage (9). Carcinoembryonic antigen (CEA) is a glycoprotein that has been evaluated in a wide range of malignancies, including breast cancer and its levels may reflect the status of disease progression or regression. Although it has been noted that CEA is less sensitive than CA15-3, it may be useful in an early diagnosis of recurrences and monitoring response to treatment (10). It has been suggested that these markers might have the potential for use in initial detection and or follow up screening for the detection of breast cancer in women (11). Consequently, the use of saliva as an adjunct diagnostic fluid has merit for further study. So the purpose of the present study was to characterize the presence of salivary C-erbB-2, CEA and CA15-3 among healthy women who use OCP to address it, as a potential confounding variable in breast carcinomas.

\section{Materials and Methods}

The participants in the present study consisted of 87 healthy women referred to the birth control center of Fatemyeh Hospital of Hamadan (the west of Iran) University of Medical Sciences from August 2008 to August 2009. The subjects were socioeconomically mixed and ranged in age from 20 to 54 years. All participants signed a standard informed consent form approved by institutional review board. A detailed questionnaire was filled out for each participant regarding her past medical history. All subjects were in good general health and were not taking any prescribed or over the counter medications (except OCP) during the course of the study. Furthermore, all subjects were premenopausal married women with normal flow and without any periods of amenorrhea. Height and weight were determined for all the subjects and then the mean body mass index (BMI) was calculated for each individual (BMI= weight/ [height]2 ). Two groups of women were selected: 1) The Case subjects who currently taking contraceptive tablets (LD tablets at ordinary dose) at least for one year. 2) Those not using any type of hormonal birth control defined as control subjects. Those subjects who have well confirmed risk factors for breast cancer were excluded from the study. These included: Family history in first and second degree relatives, age at first birth $>30$ or $<20$ years, current breast feeding, history of ionizing radiation and chemotherapy, menarche at $<12$ or $>14$ years, current smokers and alcohol consumption. Endogenous estrogen level was ignored. Two groups were matched regarding variables age, number of children, total time of breast feeding and BMI. Whole stimulated saliva was collected by standard method (12). All sample collections were done between 9:00 AM and 11:00 AM. Subjects were instructed not to drink, eat, smoke and perform oral hygiene procedures for 90 minutes before saliva collection. A standard weighted cube of paraffin gum was used by asking the person to chew at a regular rate and expectorate into a collection glass tube every one minute for a total of 5 minutes. Then the saliva samples were frozen at $-70{ }^{\circ} \mathrm{C}$ till further analysis. After collection of all samples, the saliva was analyzed for expression of biomarkers as follows: 1) C-erbB-2 concentration was determined using ELISA kit (Bender Med Systems GmbH, Vienna, Austria) and expressed as ng/ ml. 2) CEA levels were assayed by ELISA kit (Pishtaz Teb Diagnostics, Tehran, Iran) and expressed as ng/ml. 3) CA15-3 concentrations were analyzed using EIA kit (CanAg, Gothenburg, Sweden) and expressed as Units/ $\mathrm{ml}$. Biomarkers assaying was performed as recommended detailed procedures by the own manufacturers. Statistical analysis was performed by student's t-test using SPSS software package (version 13), with $\alpha$ level $<0.05$ considered as statistically significant.

\section{Results}

From 87 women participated in this study, 44 subjects were in the OCP user (Case) group and 43 in the control group. As mentioned above, some confounding variables were matched between the studied groups.

So there were no significant differences between the case and control subjects regarding age, number of children, total time of breast feeding and BMI (Table 1).

The salivary levels of C-erbB-2, CEA and CA15-3 among women with OCP exposure and their control group are shown in table 2 . The data demonstrated that there were no significant differences between the two groups in the expression of these biomarkers. 
Table 1. Characteristics of women who used OCP and those without hormonal birth control.

\begin{tabular}{lccc}
\hline $\begin{array}{l}\text { Characteristic } \\
\text { value }\end{array}$ & $\begin{array}{c}\text { OCP users }(\mathrm{n}=44) \\
\text { Mean } \pm \text { SD }\end{array}$ & $\begin{array}{c}\text { Controls }(\mathrm{n}=43) \\
\text { Mean } \pm \text { SD }\end{array}$ & P \\
\hline Age (years) & $31.4 \pm 7.13$ & $30.2 \pm 5.83$ & 0.38 \\
BMI & $24.49 \pm 4.58$ & $23.41 \pm 6.43$ & 0.36 \\
Number of children & $2.22 \pm 1.4$ & $1.67 \pm 1.3$ & 0.07 \\
Total time of breast feeding (months) & $40.19 \pm 29.37$ & $31.47 \pm 25.52$ & 0.16 \\
\hline
\end{tabular}

OPC: Oral Contraceptives

BMI: Body Mass Index

Table 2. Salivary concentrations of biomarkers in women who used OCP and those without hormonal birth control.

\begin{tabular}{lccc}
\hline Biomarkers & $\begin{array}{c}\text { OCP users }(\mathrm{n}=44) \\
\text { Mean } \pm \text { SD }\end{array}$ & $\begin{array}{c}\text { Controls }(\mathrm{n}=43) \\
\text { Mean } \pm \text { SD }\end{array}$ & P. value \\
\hline C-erb B-2 (ng/ml) & $1.93 \pm 0.95$ & $1.70 \pm 0.96$ & 0.26 \\
CA 15-3 (units $/ \mathrm{ml})$ & $12.58 \pm 9.79$ & $16.19 \pm 10.8$ & 0.44 \\
CEA (ng/ml) & $34.46 \pm 17.41$ & $31.62 \pm 17.24$ & 0.44 \\
\hline
\end{tabular}

OPC: Oral Contraceptives

\section{Discussion}

Different studies have defined the risk of breast cancer by evaluation of tumor markers expression and the use of OCP; nevertheless some have reported no significant correlation (13-15). Overall, there is a mutual agreement on the usefulness of tumor markers (e.g. C-erbB-2, CEA and CA15-3) for the prognosis of breast cancer (16-18). In the present study, all participants were similar according to the marital status, menstrual cycle, menopause status, onset of menarche and the age of first birth. Also, variables age, number of children, total time of breast feeding and BMI were matched between OCP and non-OCP users. A cohort study among healthy women showed no association between variables age, race, tobacco usage, menopausal status, estrogen use, systemic diseases, prescription medications and salivary C-erbB-2 and CA15-3 (11). Streckfus et al. (8) suggested that the assay of salivary C-erbB-2 is reliable and might have potential use in the initial detection and follow up screening for breast cancer. The two aforementioned studies have utilized stimulated saliva and ELISA method for assaying of tumor markers among healthy women. The results of the present study indicated that there were no statisti- cal differences between OCP users and the controls in the salivary expression of biomarkers. McIntyre et al . (2) have analyzed stimulated whole saliva samples of 40 healthy women (OCP and non-OCP users) regarding the expression of C-erbB-2 and CA15-3 using immuno assaying method. They selected premenopausal women with normal menstrual cycle and finally found no significant differences between the control and study subjects. Moreover, they have noted that anti-estrogen therapy (e.g. tamoxifen) should not alter the expression of these biomarkers. Although the findings of McIntyre et al (2) and the present study are similar, the results of the present study are more clear and definite. In a review study by Hankinson et al. (15) the well confirmed risk factors for breast cancer were as follow: Family history of breast cancer, increase of BMI, benign breast diseases, not parity, age at first birth $>30$ or $<20$ years, no lactation, menopause at $>54$ or $<45$ years, high endogenous estrogen levels, history of ionizing radiation exposure, menarche at $<12$ or $>14$ years and alcohol consumption. Approximately all mentioned risk factors were considered in the case and control groups of the present study. Furthermore, a larger sample size was analyzed based 
on the statistical reasons to find more obvious relation between OCP usage and the expression of tumor markers. The results of the present study demonstrated that the monitoring of women with high estrogen levels using salivary C-erbB-2, CEA and CA15-3 seems not to be useful, however further definitive studies are mandatory. Interest in saliva even more increased with the finding that saliva is filled with hundreds of components that may serve to detect systemic diseases or evidence of exposure to various harmful substances, as well as provide biomarkers of health and disease status (19). In our study, saliva samples collection was done under the standard method defined by Navazesh (12). It has been noted; although saliva is potentially an attractive diagnostic fluid, standardization of the collection procedure is of ultimate importance in order to obtain reproducible results. Standardization of the conditions for collection of saliva is strictly essential for achieving reliability and interpretation of the data (20). Saliva as a diagnostic medium has many advantages over serum and many of the hazards associated with blood collection do not apply to saliva. It is a clear colorless fluid that its collection is easy and noninvasive and may be done repeatedly without discomfort to the patient. There is no need for sharps, which have the potential for cross contamination among patients when used improperly and present a danger to health care personnel. Because of the low concentrations of antigens in saliva, HIV and hepatitis infections are much less of a danger from saliva than from blood $(2,9,11,19)$. In summery; saliva could be a cost effective tool to monitor the health status, screening and diagnostic approaches.

\section{References}

References with links to Crossref - DOI

1. Westhoff CL, Heartwell S, Edwards S, Zieman M, Stuart G, Cwiak $\mathrm{C}$, et al. Oral contraceptive discontinuation: do side effects matter? Am J Obstet Gynecol. 2007;196:412.e1-6.

2. McIntyre R, Bigler L, Dellinger T, Pfeifer M, Mannery T, Streckfus C. Oral contraceptive usage and the expression of CA 15-3 and c-erbB-2 in the saliva of healthy women. Oral Surg Oral Med Oral Pathol Oral Radiol Endod. 1999;88:687-90.

3. Biglia N, Mariani L, Sgro L, Mininanni P, Moggio G, Sismondi P. Increased incidence of lobular breast cancer in women treated with hormone replacement therapy: implications for diagnosis, surgical and medical treatment. Endocr Relat Cancer. 2007;14:549-67.

4. Lester J. Breast cancer in 2007: incidence, risk assessment, and risk reduction strategies. Clin J Oncol Nurs. 2007;11:619-22.

5. Emekli-Alturfan E, Demir G, Kasikci E, Tunali-Akbay T, Pisiriciler R, Caliskan E, et al. Altered biochemical parameters in the saliva of patients with breast cancer. Tohoku J Exp Med. 2008;214:89-96.

6. Ross JS, Fletcher JA. The HER-2/neu Oncogene in Breast Cancer: Prognostic Factor, Predictive Factor, and Target for Therapy. Oncologist. 1998;3:237-52.

7. Streckfus CF, Bigler L, Dellinger T, Kuhn M, Chouinard N, Dai X. The expression of the c-erbB-2 receptor protein in glandular salivary secretions. J Oral Pathol Med. 2004;33:595-600.

8. Streckfus C, Bigler L, Dellinger T, Dai X, Cox WJ, McArthur A, et al. Reliability assessment of soluble c-erbB-2 concentrations in the saliva of healthy women and men. Oral Surg Oral Med Oral Pathol Oral Radiol Endod. 2001;91:174-9.
9. Agha-Hosseini F, Mirzaii-Dizgah I, Rahimi A. Correlation of serum and salivary CA15-3 levels in patients with breast cancer. Med Oral Patol Oral Cir Bucal. 2009;14:e521-4.

10. Guadagni F, Ferroni P, Carlini S, Mariotti S, Spila A, Aloe S, et al. A re-evaluation of carcinoembryonic antigen (CEA) as a serum marker for breast cancer: a prospective longitudinal study. Clin Cancer Res. 2001;7:2357-62.

11. Streckfus C, Bigler L, Dellinger T, Pfeifer M, Rose A, Thigpen JT. CA 15-3 and c-erbB-2 presence in the saliva of women. Clin Oral Investig. 1999;3:138-43.

12. Navazesh M; ADA Council on Scientific Affairs and Division of Science. How can oral health care providers determine if patients have dry mouth? J Am Dent Assoc. 2003;134:613-20.

13. Dumeaux V, Fournier A, Lund E, Clavel-Chapelon F. Previous oral contraceptive use and breast cancer risk according to hormone replacement therapy use among postmenopausal women. Cancer Causes Control. 2005;16:537-44.

14. Kahlenborn C, Modugno F, Potter DM, Severs WB. Oral contraceptive use as a risk factor for premenopausal breast cancer: a metaanalysis. Mayo Clin Proc. 2006;81:1290-302.

15. Hankinson SE, Colditz GA, Willett WC. Towards an integrated model for breast cancer etiology: the lifelong interplay of genes, lifestyle, and hormones. Breast Cancer Res. 2004;6:213-8.

16. Molina R, Filella X, Zanon G, Pahisa J, Alicarte J, Munoz M, et al. Prospective evaluation of tumor markers (c-erbB-2 oncoprotein, CEA and CA 15.3) in patients with locoregional breast cancer. Anticancer Res. 2003;23:1043-50.

17. Molina R, Jo J, Filella X, Zanón G, Farrus B, Muñoz M, et al. CerbB-2, CEA and CA 15.3 serum levels in the early diagnosis of recurrence of breast cancer patients. Anticancer Res. 1999;19:2551-5.

18. Molina R, Jo J, Filella X, Zanon G, Pahisa J, Munoz M, et al. C-erbB-2 oncoprotein, CEA, and CA 15.3 in patients with breast cancer: prognostic value. Breast Cancer Res Treat. 1998;51:109-19.

19. Schipper RG, Silletti E, Vingerhoeds MH. Saliva as research material: biochemical, physicochemical and practical aspects. Arch Oral Biol. 2007;52:1114-35.

20. Nieuw Amerongen AV, Ligtenberg AJ, Veerman EC. Implications for diagnostics in the biochemistry and physiology of saliva. Ann N Y Acad Sci. 2007;1098:1-6.

\section{Acknowledgments}

This study was approved and financially supported by Hamadan University of Medical Sciences. We are also grateful to Dr M. Hajilooi for his expertise in performing the immunological assays and Mr. Mani Kashani for his valuable help in statistical analysis. 